\title{
Sobolev inequalities, rearrangements, isoperimetry and interpolation spaces
}

\author{
Joaquim Martín* and Mario Milman \\ Dedicated to our friends Björn Jawerth and Evgeniy Pustylnik on the ocassion of their 130th \\ birthday (57th and 73th birthdays, respectively).
}

\begin{abstract}
We characterize Poincaré inequalities in metric spaces using rearrangement inequalities.
\end{abstract}

\section{Introduction}

Our starting point is the classical Gagliardo-Nirenberg inequality which states that, for $n>1, \frac{1}{n^{\prime}}=1-\frac{1}{n}$,

$$
\|f\|_{n^{\prime}} \leq \tau_{n}^{-1}\||\nabla f|\|_{L^{1}}, f \in \operatorname{Lip}_{0}\left(\mathbb{R}^{n}\right)
$$

where $\operatorname{Lip}_{0}\left(\mathbb{R}^{n}\right)$ denotes the set of Lipschitz function on $\mathbb{R}^{n}$ with compact support, $\tau_{n}=n \beta_{n}^{1 / n}$ and $\beta_{n}=$ volume of the unit ball in $\mathbb{R}^{n}$. It is well known (cf. [21] and [11), that (1.1) is equivalent to the isoperimetric inequality]: for all Borel sets $A$ with $m(A)<\infty$, we have

$$
\tau_{n}(m(A))^{1 / n^{\prime}} \leq m^{+}(A) .
$$

We argue that it is worthwhile to consider a slightly more general problem. Let $X=X\left(\mathbb{R}^{n}\right)$ be a rearrangement invariant spacel: We ask for necessary and sufficient conditions such that

$$
\|f\|_{X} \leq c\||\nabla f|\|_{L^{1}}, f \in \operatorname{Lip}_{0}\left(\mathbb{R}^{n}\right)
$$

holds. Maz'ya's classical method already shows that the problem has a remarkably simple solution: (1.3) holds if and only if there exists a constant $c=c(n)>0$ such that for all Borel sets $A$ with $m(A)<\infty$,

$$
\phi_{X}(m(A)) \leq \mathrm{cm}^{+}(A),
$$

Key words and phrases. Sobolev inequalities, Poincaré, symmetrization, isoperimetric inequalities, interpolation.

*Partially supported in part by Grants MTM2007-60500, MTM2008-05561-C02-02.

This paper is in final form and no version of it will be submitted for publication elsewhere.

2000 Mathematics Subject Classification Primary: 46E30, 26D10.

${ }^{1}$ Here $m$ stands for Lebesgue measure and $m^{+}$for Minkowski's content.

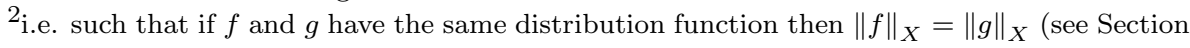
2.4 below). 
where $\phi_{X}(t)$ is the fundamental function ${ }^{3}$ of $X$ :

$$
\phi_{X}(t)=\left\|\chi_{A}\right\|_{X}, \text { with } m(A)=t .
$$

Formally (abusing the notation), the implication (1.3) $\Rightarrow(1.4)$ follows inserting " $f=\chi_{A}$ " in (1.3) and then computing $\|\nabla f\|_{L_{1}}=m^{+}(A),\|f\|_{X}=\phi_{X}(m(A))$.

We now consider the converse statement. Here it will become clear why we insist to work within the class of rearrangement invariant spaces: Indeed, if we fix before hand a specific subclass of rearrangement invariant spaces (e.g. Orlicz spaces) we would miss a remarkable self-improving phenomenon.

Let $f \in \operatorname{Lip}_{0}\left(\mathbb{R}^{n}\right)$, and let $A_{t}=\{|f|>t\}, m\left(A_{t}\right)=m_{f}(t)$ (= the distribution function of $f$ ), then, from (1.4), and the co-area formula, we find that

$$
\begin{aligned}
\int_{0}^{\infty} \phi_{X}\left(m_{f}(t)\right) d t & \leq c \int_{0}^{\infty} m^{+}\left(A_{t}\right) d t=c \int_{\mathbb{R}^{n}}|\nabla| f|(x)| d x \\
& \leq c \int_{\mathbb{R}^{n}}|\nabla f(x)| d x .
\end{aligned}
$$

The integral on the left hand side is, by definition, the norm of $f$ in the Lorentz space $\Lambda(X)$ associated with $X$,

$$
\|f\|_{\Lambda(X)}=\int_{0}^{\infty} \phi_{X}\left(m_{f}(t)\right) d t
$$

$\Lambda(X)$ is contained (and, in general, strictly contained) in $X$; in other words we have (cf. [5])

$$
\|f\|_{X} \leq\|f\|_{\Lambda(X)} .
$$

Altogether, we have thus shown that

$$
\|f\|_{X} \leq\|f\|_{\Lambda(X)} \leq c\|\nabla f\|_{L^{1}} .
$$

Therefore, for $f \in \operatorname{Lip}_{0}\left(\mathbb{R}^{n}\right)$, we have the remarkable self improvement

$$
\|f\|_{X} \leq c\||\nabla f|\|_{L^{1}} \Leftrightarrow\|f\|_{\Lambda(X)} \leq c\||\nabla f|\|_{L^{1}} .
$$

But we are not quite done yet. We could have obtained the same result starting from a much weaker inequality. Indeed, there is another natural rearrangement invariant space (r.i. space) associated to $X$ : the somewhat larger Marcinkiewicz space $M(X)$ (=Marcinkiewicz=weak type space) defined by the quasi-norm

$$
\|f\|_{M(X)}=\sup _{t>0} f^{*}(t) \phi_{X}(t)=\sup _{t>0} t \phi_{X}\left(m_{f}(t)\right),
$$

where $f^{*}$ is the non-increasing rearrangement] of $f$. The fundamental functions of these spaces satisfy

$$
\phi_{M(X)}(t)=\phi_{\Lambda(X)}(t)=\phi_{X}(t)
$$

It follows that for $f \in \operatorname{Lip}_{0}\left(\mathbb{R}^{n}\right)$,

$$
\|f\|_{M(X)} \leq c\||\nabla f|\|_{L^{1}} \Leftrightarrow\|f\|_{\Lambda(X)} \leq c\||\nabla f|\|_{L^{1}} .
$$

\footnotetext{
${ }^{3}$ It is well known and easy to see that $\phi_{X}$ is continous, increasing and equivalent to a concave function.

${ }^{4} f^{*}$ is the generalized inverse of $m_{f}$.
} 
These self-improving results are best possible since the spaces $\Lambda(X), M(X)$ are respectively the smallest and largest r.i. spaces with fundamental functions equal to $\phi_{X}(t)$ (cf. (1.6)), and such that (cf. [5])

$$
\Lambda(X) \subset X \subset M(X) .
$$

A consequence of our discussion is that the optimal spaces $X$ for the embedding (1.3) must be Lorentz spaces.

We now develop a quantitative connection with Euclidean isoperimetry. For this purpose it is important to consider the isoperimetric profile of $\mathbb{R}^{n}$

$$
I(t)=\inf _{m(A)=t} m^{+}(A) .
$$

The isoperimetric inequality (1.2) is the statement that for $n>1, I(t)$ is given by

Note that $\Lambda\left(L^{n^{\prime}}\right)=L\left(n^{\prime}, 1\right)$ :

$$
I(t)=\tau_{n} t^{1 / n^{\prime}}, \quad n^{\prime}=n /(n-1) .
$$

$$
\begin{aligned}
\|f\|_{\Lambda\left(L^{n^{\prime}}\right)} & =\int_{0}^{\infty}\left(m_{f}(t)\right)^{1 / n^{\prime}} d t=\int_{0}^{\infty} t^{1 / n^{\prime}} d f^{*}(t) \\
& =\frac{1}{n^{\prime}} \int_{0}^{\infty} t^{1 / n^{\prime}} f^{*}(t) \frac{d t}{t}=\frac{1}{n^{\prime}}\|f\|_{L\left(n^{\prime}, 1\right)} .
\end{aligned}
$$

Therefore the previous analysis shows that the Gagliardo-Nirenberg inequality (1.1) self improves to its sharper form

$$
\|f\|_{L\left(n^{\prime}, 1\right)} \leq n^{\prime} \tau_{n}^{-1}\||\nabla f|\|_{L^{1}} .
$$

The results that underlie the narrative above, including the sharp GagliardoNirenberg inequality (1.7), are, of course, well known. But the added generality becomes more illuminating when we move away from the classical Euclidean setting. Indeed, the argument that gives the equivalence (1.3) $\Leftrightarrow(1.4)$ is very general and holds replacing $\mathbb{R}^{n}$ by fairly general metric measure spaces as long as we have a suitable co-area formula (cf. Bobkov-Houdré [6], Coulhon $[\mathbf{9}$ and the references therein).

Consider a connected, metric, non-atomic measure space $(\Omega, d, \mu)$. For a Lipschitz function $f$ on $\Omega$ we let $|\nabla f(x)|=\lim \sup _{d(x, y) \rightarrow 0} \frac{|f(x)-f(y)|}{d(x, y)}$, and let $\operatorname{Lip}_{0}(\Omega)$ denote the Lipschitz functions with compact support. Let us further assume that the equivalence between

$$
\|f\|_{X} \leq c\||\nabla f|\|_{L^{1}}, \quad f \in \operatorname{Lip}_{0}(\Omega)
$$

and

$$
\phi_{X}(\mu(A)) \leq c \mu^{+}(A),
$$

hold 5 . We suppose, moreover, that the associated isoperimetric profile $I=I_{\Omega}$, defined by

$$
I(t)=\inf _{\mu(A)=t} \mu^{+}(A)
$$

is continuous, increasing and concave. The same analysis then shows that the best possible r.i. space such that (1.8) holds is a Lorentz space and its corresponding fundamental function $\phi$, say, must be such that (1.9) holds. The optimal space

\footnotetext{
${ }^{5}$ here $\mu(A)<\infty, \mu^{+}(A)=$ perimeter of $A$ (see Section 2.1 below).
} 
corresponds to choosing the largest possible $\phi$ that satisfies (1.9), consequently the best choice is $\phi=I=I_{\Omega}$ ! Therefore we have

$$
\|f\|_{\Lambda(I)} \leq\||\nabla f|\|_{L^{1}},
$$

where $\Lambda(I)$ is "the isoperimetric Lorentz space" defined by

$$
\|f\|_{\Lambda(I)}=\int_{0}^{\infty} I\left(\mu_{f}(t)\right) d t .
$$

General Sobolev inequalities, including Logarithmic Sobolev inequalities, fit into this picture very naturally. Indeed, in this fashion we have a natural method to construct best possible Sobolev inequalities if we understand the isoperimetry associated with a given geometry.

It is worthwhile to discuss in some detail how this point of view applies to Gaussian measure (cf. [18). In the Gaussian world the isoperimetric function $I$ has the following properties: $I$ is defined on $[0,1]$, it is increasing on $[0,1 / 2]$, symmetric about $1 / 2$, and $I$ is concave. Since we are dealing with a probability space, from the point of view of describing the underlying function spaces it is only important to know the behavior of $I$ near the origin. We actually have 6

$$
I(t) \simeq t\left(\log \frac{1}{t}\right)^{1 / 2}, \text { for } t \in[0,1 / 2] .
$$

In this case (1.11) is not a norm but nevertheless the set of all $f$ with $\|f\|_{\Lambda(I)}<\infty$ is equivalent to the Lorentz space $L(\log L)^{1 / 2}$ : In other words, as sets,

$$
\Lambda(I)=L(\log L)^{1 / 2} \text {. }
$$

In this setting the inequality (1.10), which is due to Ledoux [16, can be seen as part of the usual family of Log Sobolev inequalities. Thus, in the Gaussian world, Ledoux's inequality plays the role of the classical (Euclidean) sharp GagliardoNirenberg inequality.

More generally, the "isoperimetric Lorentz spaces" can be used to construct the corresponding Gagliardo-Nirenberg inequalities in other geometries.

Let us mention two obvious drawbacks of the previous discussion: (a) we only considered Sobolev spaces where the gradient is in $L^{1}$, (b) the analysis is *space dependent*. On the other hand, already in the Euclidean case, Maz'ya showed that "all $L^{p}$ Sobolev" inequalities can be obtained from the isoperimetric inequality or, equivalently, from (1.1). In our recent work we have considered the extension of Maz'ya's ideas to rearrangement invariant spaces.

Maz'ya's smooth truncation method has been extensively studied in the literature (cf. 11, 13, and the references therein) but in our development we required an extension that leads to pointwise rearrangement 1 inequalities that depend on the isoperimetric profile. For example, we showed in a very general setting (cf. 17, [18, [19]) inequalities of the form

$$
f_{\mu}^{* *}(t)-f_{\mu}^{*}(t) \leq \frac{t}{I(t)}|\nabla f|_{\mu}^{* *}(t), f \in \operatorname{Lip}(\Omega) \cap L^{1}(\Omega),
$$

\footnotetext{
${ }^{6}$ Here the symbol $f \simeq g$ indicates the existence of a universal constant $c>0$ (independent of all parameters involved) such that $(1 / c) f \leq g \leq c f$. Likewise the symbol $f \preceq g$ will mean that there exists a universal constant $c>0$ (independent of all parameters involved) such that $f \leq c g$.

7 also called "symmetrization" inequalities since they are often expressed in terms of "symmetric" rearrangements.
} 
where $f_{\mu}^{* *}(t)=\frac{1}{t} \int_{0}^{t} f_{\mu}^{*}(s) d s$, and $f_{\mu}^{*}$ is the non increasing rearrangement of $f$ with respect to the measure $\mu$ on $\Omega$ (see Section 2.4 below). Let us now show in some detail that (1.12) implies the isoperimetric inequality (cf. [19]). Following [6] we select a sequence $\left\{f_{n}\right\}_{n \in N}$ in $\operatorname{Lip}(\Omega) \cap L^{1}(\Omega)$, such that $f_{n} \underset{L^{1}}{\rightarrow} \chi_{A}$, and

$$
\mu^{+}(A) \geq \lim \sup _{n \rightarrow \infty}\left\|\left|\nabla f_{n}\right|\right\|_{L^{1}} .
$$

Let $t>\mu(A)$ and apply (1.12) to this sequence. We have

$$
\left(f_{n}\right)_{\mu}^{* *}(t)-\left(f_{n}\right)_{\mu}^{*}(t) \leq \frac{t}{I(t)}\left|\nabla f_{n}\right|_{\mu}^{* *}(t), n \in N
$$

By definition

$$
\begin{aligned}
t\left|\nabla f_{n}\right|_{\mu}^{* *}(t) & =\int_{0}^{t}\left|\nabla f_{n}\right|_{\mu}^{*}(s) d s \\
& \leq\left\|\left|\nabla f_{n}\right|\right\|_{L^{1}} .
\end{aligned}
$$

Therefore,

$$
\lim \sup _{n} t\left|\nabla f_{n}\right|_{\mu}^{* *}(t) \leq \lim _{n \rightarrow \infty} \sup _{n \rightarrow \infty}\left\|\left|\nabla f_{n}\right|\right\|_{L^{1}} \leq \mu^{+}(A) .
$$

On the other hand by $[\mathbf{1 2}$ we have

$$
I(t)\left(\left(f_{n}\right)_{\mu}^{* *}(t)-\left(f_{n}\right)_{\mu}^{*}(t)\right) \rightarrow I(t)\left(\chi_{A}^{* *}(t)-\chi_{A}^{*}(t)\right) .
$$

Combining our findings we have

$$
I(t)\left(\chi_{A}^{* *}(t)-\chi_{A}^{*}(t)\right) \leq \mu^{+}(A), \text { for all } t>\mu(A) .
$$

Now, since $\chi_{A}^{*}=\chi_{(0, \mu(A))}$, we have that for $t>\mu(A)$,

$$
\chi_{A}^{*}(t)=\chi_{(0, \mu(A))}(t)=0, \chi_{A}^{* *}(t)=\frac{1}{t} \int_{0}^{t} \chi_{(0, \mu(A))}(s) d s=\frac{\mu(A)}{t} .
$$

Inserting this information in (1.14) we get

$$
I(t) \frac{\mu(A)}{t} \leq \mu^{+}(A) \text {. }
$$

Finally we let $t \rightarrow \mu(A)$; then, by the continuity of $I$, we obtain the isoperimetric inequality

$$
I(\mu(A)) \leq \mu^{+}(A)
$$

We now discuss the corresponding Sobolev inequalities with $L^{q}, q>1$, replacing the $L^{1}$ norm on the right hand side of (1.8). Again we shall work on suitable metric probability spaces $(\Omega, d, \mu) \sqrt{8}$, and we consider Poincaré inequalities of the form

$$
\|f-m(f)\|_{X} \leq c\||\nabla f|\|_{L^{q}}, f \in \operatorname{Lip}(\Omega), q>1,
$$

where $X$ is a r.i. space and $m(f)$ is a mediar 9 of $f$. As is well known, inequalities of this type can be characterized using Maz'ya's theory of capacities (cf. [23]). The weak type version of (1.15) reads:

$$
\|f-m(f)\|_{M(X)} \preceq\||\nabla f|\|_{L^{q}}, f \in \operatorname{Lip}(\Omega), q>1 .
$$

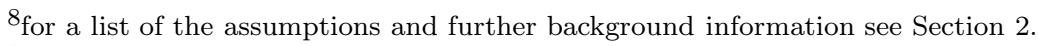

9 a real number $m(f)$ such that $\mu\{f \geq m(f)\} \geq 1 / 2$ and $\mu\{f \leq m(f)\} \geq 1 / 2$.
} 
In this context a result of E. Milman [24, Proposition 3.8] can be rewritten in our notation as saying that (1.16) is equivalent to

$$
\left(\phi_{X}(t)\right)^{q} \preceq \operatorname{cap}_{q}(t, 1 / 2), 0<t<1 / 2,
$$

where (using temporarily $[10$ the definition of $[\mathbf{7}$ rather than the one in [24])

$$
\operatorname{cap}_{q}(t, 1 / 2)=\inf \left\{\||\nabla \Phi|\|_{L^{q}}^{q}: \mu\{\Phi=1\} \geq t, \mu\{\Phi=0\} \geq 1 / 2\right\},
$$

and the infimum is taken over all $\Phi: \Omega \rightarrow[0,1]$ that are Lipschitz on balls.

To relate (1.17) to $X$ norm inequalities we use the $\Lambda_{q}(X)$ Lorentz spaces defined by

$$
\|f\|_{\Lambda_{q}(X)}=\left(\int_{0}^{\infty} \phi_{X}\left(\mu_{f}(t)\right) d t^{q}\right)^{1 / q} .
$$

We say that $X$ is $q$-concave if the space $X_{(q)}$ defined by:

$$
X_{(q)}=\left\{f:|f|^{1 / q} \in X\right\}, \quad\|f\|_{X_{(q)}}=\left\||f|^{1 / q}\right\|_{X}^{q},
$$

is a r.i. space (see Section 2.4 below). Moreover, it follows from the definitions that

$$
\|f\|_{X}^{q}=\left\||f|^{q}\right\|_{X_{(q)}} .
$$

We develop this theme for the $\Lambda_{q}(X)$ scale in detail. From $\mu_{|f|^{q}}(t)=\mu_{|f|}\left(t^{1 / q}\right)$, we see that 11

$$
\left\||f|^{q}\right\|_{\Lambda(X)}=\|f\|_{\Lambda_{q}(X)}^{q}
$$

in particular for $X_{(q)}$ we have

$$
\left\||f|^{q}\right\|_{\Lambda\left(X_{(q)}\right)}=\|f\|_{\Lambda_{q}\left(X_{(q)}\right)}^{q} .
$$

Note that for any measurable set with $\mu(A)=t$, we have

$$
\phi_{X_{(q)}}(t)=\left\|\chi_{A}\right\|_{X_{(q)}}=\left\|\chi_{A}\right\|_{X}^{q}=\left(\phi_{X}(t)\right)^{q},
$$

in particular if $X$ is $q$-concave the function $\phi_{X_{(q)}}(t)=\left(\phi_{X}(t)\right)^{q}$ is concave, and (1.17) now reads

$$
\phi_{X_{(q)}}(t) \preceq \operatorname{cap}_{q}(t, 1 / 2), 0<t<1 / 2 .
$$

Thus, using the characterization of Sobolev norms in terms of capacities, due to Maz'ya (in the form given by Bobkov and Zegarlinski for metric paces [7, Lemma $5.6]$ ), we now show that (1.16) self improves to

$$
\|f-m(f)\|_{\Lambda_{q}\left(X_{(q)}\right)} \preceq\||\nabla f|\|_{L^{q}} .
$$

To see this we use (1.20) as follows. First we observe that it is enough to prove (1.21) for positive functions that are Lipschitz on balls, such that $\|f\|_{\infty} \leq 1$, and, moreover, such that $m(f)=0$ (see details of the argument that proves this assertion in [24, page 331]). Let $f$ be a function satisfying all these conditions, then, by (1.20), we have

$$
\phi_{X_{(q)}}\left(\mu_{f}(t)\right) \preceq \operatorname{cap}_{q}\left(\mu_{f}(t), 1 / 2\right) .
$$

\footnotetext{
${ }^{10}$ See Definition 1 below.

${ }^{11}$ in other words $\Lambda_{q}(X)_{(q)}=\Lambda(X)$.
} 
Therefore

$$
\begin{aligned}
\|f-0\|_{\Lambda_{q}\left(X_{(q)}\right)}^{q} & =\int_{0}^{\infty} \phi_{X_{(q)}}\left(\mu_{f}(t)\right) d t^{q} \preceq \int_{0}^{\infty} \operatorname{cap}_{q}\left(\mu_{f}(t), 1 / 2\right) d t^{q} \\
& \preceq\|\nabla f \mid\|_{L^{q}}^{q},
\end{aligned}
$$

where the last inequality follows from Bobkov and Zegarlinski [7, Lemma 5.6] changing 2 for $q$ in the argument given there.

Finally, combining with (2.1), (1.18) and (1.19), we obtain

$$
\begin{aligned}
\|f\|_{X}^{q} & =\left\||f|^{q}\right\|_{X_{(q)}} \\
& \leq\left\||f|^{q}\right\|_{\Lambda\left(X_{(q)}\right)} \\
& =\|f\|_{\Lambda_{q}\left(X_{(q)}\right)}^{q} \\
& \preceq\|\mid \nabla f\|_{L^{q}}^{q} .
\end{aligned}
$$

Thus, we see that the Sobolev self improvement that we obtained in the case $q=1$ extends to the case $q>1$, but now it is expressed in terms of the $\Lambda_{q}\left(X_{(q)}\right)$ spaces. More precisely, for $q$ - concave spaces we have the following equivalences on Lip functions

$$
\begin{aligned}
&\|f-m(f)\|_{M(X)} \preceq\||\nabla f|\|_{L^{q}} \Leftrightarrow\|f-m(f)\|_{\Lambda_{q}\left(X_{(q)}\right)} \preceq\||\nabla f|\|_{L^{q}} \\
& \Leftrightarrow\|f-m(f)\|_{X} \preceq\||\nabla f|\|_{L^{q}} .
\end{aligned}
$$

After this lengthy introduction we now describe the purpose of this note. We shall consider the analogues of the rearrangement inequalities (1.12) that correspond to consider homogenous Sobolev norms with $q>1$ on the right hand side. The inequalities we shall obtain will be naturally formulated in terms of the $q$-convexification $X^{(q)}$ of $X$ (see (2.23) in Section 2.4 below). We also pay close attention to the basic assumptions that one needs to place on the isoperimetric profile, and the probability measure spaces, in order to develop a meaningful theory with mild assumptions. In particular, we are able to extend some results of [19] under weaker assumptions.

Finally in Section 3 we shall briefly discuss a connection with interpolation theory, that was recently developed in $\mathbf{1 0}$, that shows a larger context for the Sobolev oscillation inequalities and connects some aspects of our work with the theory of extrapolation of martingale inequalities.

\section{Capacitary Inequalities}

2.1. Background. From now on "a metric probability space $(\Omega, d, \mu)$ " will be a connected separable metric space $(\Omega, d, \mu)$ equipped with a non-atomic Borel probability measure $\mu$. For measurable functions $u: \Omega \rightarrow \mathbb{R}$, the distribution function of $u$ is given by

$$
\mu_{u}(t)=\mu\{x \in \Omega:|u(x)|>t\} \quad(t>0) .
$$

The decreasing rearrangement $u_{\mu}^{*}$ of $u$ is the right-continuous non-increasing function from $(0,1)$ to $[0, \infty]$ which is equimeasurable with $u$. Namely,

$$
u_{\mu}^{*}(s)=\inf \left\{t \geq 0: \mu_{u}(t) \leq s\right\} .
$$


We have (cf. [5]),

$$
\sup _{\mu(E) \leq t} \int_{E}|u(x)| d \mu(x)=\int_{0}^{\mu(E)} u_{\mu}^{*}(s) d s .
$$

Since $u_{\mu}^{*}$ is decreasing, the function $u_{\mu}^{* *}$, defined for integrable functions by

$$
u_{\mu}^{* *}(t)=\frac{1}{t} \int_{0}^{t} u_{\mu}^{*}(s) d s
$$

is also decreasing and, moreover,

$$
u_{\mu}^{*} \leq u_{\mu}^{* *} .
$$

As customary, if $A \subset \Omega$ is a Borel set, the perimeter or Minkowski content of $A$ is defined by

$$
\mu^{+}(A)=\lim \inf _{h \rightarrow 0} \frac{\mu\left(A_{h}\right)-\mu(A)}{h},
$$

where $A_{h}=\{x \in \Omega: d(x, A)<h\}$.

The isoperimetric profile $I_{(\Omega, d, \mu)}$ is defined as the pointwise maximal function $I_{(\Omega, d, \mu)}:[0,1] \rightarrow[0, \infty)$ such that

$$
\mu^{+}(A) \geq I_{(\Omega, d, \mu)}(\mu(A))
$$

holds for all Borel sets $A$.

For a Lipschitz function $f$ on $\Omega$ (briefly $f \in \operatorname{Lip}(\Omega)$ ) we define, as usual, the modulus of the gradient by

$$
|\nabla f(x)|=\lim \sup _{d(x, y) \rightarrow 0} \frac{|f(x)-f(y)|}{d(x, y)} .
$$

One of the themes of our recent paper [19] was to characterize generalized Gagliardo-Nirenberg inequalities and Poincaré inequalities using rearrangement inequalities. The setting of [19] were metric probability spaces $(\Omega, d, \mu)$ that satisfy the following conditions:

Condition 1: The isoperimetric profile $I_{(\Omega, d, \mu)}$ is a concave continuous function, increasing on $(0,1 / 2)$, symmetric about the point $1 / 2$ such that, moreover, vanishes at zero.

Remark 1. Condition 1 played an important role in the formulation of the inequalities obtained in $\mathbf{1 9}$. In this note we shall show that, suitably reformulated (cf. 2.9 below), our inequalities remain true under the weaker Condition 1' below.

Condition 2: For every $f \in \operatorname{Lip}(\Omega)$, and every $c \in R$, we have that $|\nabla f(x)|=$ $0, \mu$-a.e. on the set $\{x: f(x)=c\}$.

Remark 2. Condition 2 is used to compare the gradients of Lip functions that coincide on a given set, which is particularly useful to deal with truncations. Moreover, it implies that $\int_{\{f=t\}}|\nabla f| d \mu=0$, even on sets where may have $\mu\{f=t\}>0$. Using an approximation argument of E. Milman [26. Remark 3.3] we will show how to dispense with this condition as well (cf. Theorem 0 below).

In this paper, we consider Sobolev inequalities for $q \geq 1$, moreover, following a suggestion of Michel Ledoux, we shall impose weaker restrictions on the metric spaces. More specifically, we will eliminate Condition 2 and replace Condition 1 with the following much weaker assumption 
Condition 1': The isoperimetric profile $I_{(\Omega, d, \mu)}$ is a positive continuous function that vanishes at zero.

Remark 3. Notice that the continuity assumption, and 2.4), (2.2) below, imply that I is symmetric about the point $1 / 2$ (see [25. Corollary 6.5]). Moreover, we see that for $q>1$ the function $\frac{1}{\left(\inf _{t \leq z \leq 1 / 2} I(z)\right)^{\frac{q}{q-1}}}$ is locally integrable on $(0,1)$.

The notion of capacity plays a fundamental role in the theory developed by $\mathrm{V}$. G. Maz'ya and his school to study functional inequalities and embedding theorems (see [23]). For the study of capacities in metric spaces we also refer to see [2], 7], 24, and the references therein). Capacities will also play a decisive role in our development in this note.

Definition 1. Let $(\Omega, d, \mu)$ be a metric probability space, and let $1 \leq q<\infty$. Given two Borel sets $A \subset B \subset \Omega$, the q-capacity of $A$ relative to $B$ is defined by

$$
\operatorname{Cap}_{q}(A, B)=\inf \left\{\||\nabla \Phi|\|_{L^{q}}: \Phi_{\mid A}=1, \Phi_{\mid \Omega \backslash B}=0\right\},
$$

where the infimum is over all $\Phi: \rightarrow[0,1]$ which are Lipschitz-on-balls.

Let $0<a \leq b<1$, the $q$-capacity profile is defined by

$$
\begin{aligned}
\operatorname{cap}_{q}(a, b) & =\inf \left\{\operatorname{Cap}_{q}(A, B): A \subset B, \quad \mu\{A\} \geq a, \mu\{B\} \leq b\right\} \\
& =\inf \left\{\||\nabla \Phi|\|_{L^{q}}: \mu\{\Phi=1\} \geq a, \mu\{\Phi=0\} \geq 1-b\right\},
\end{aligned}
$$

where the latter infimum is taken over all $\Phi: \rightarrow[0,1]$ which are Lipschitz-on-balls.

Let us also recall some properties concerning capacities that will be useful in what follows:

(1) It is plain from the definition that

$$
\operatorname{cap}_{q}(a, b)=\operatorname{cap}_{q}(1-b, 1-a), \quad(0<a \leq b<1) .
$$

Moreover, the functional $a \rightarrow \operatorname{cap}_{q}(\cdot, b)$ is increasing; and $b \rightarrow \operatorname{cap}_{q}(a, \cdot)$ is decreasing.

(2) (See [23, p. 105] and [24]) Let $1<q<\infty$, then

$$
\frac{1}{\operatorname{cap}_{q}(a, b)} \leq\left(\int_{a}^{b} \frac{d s}{\operatorname{cap}_{1}(s, b)^{\frac{q}{q-1}}}\right)^{\frac{q-1}{q}}, \quad(0<a \leq b<1) .
$$

(3) (See [22, [11, 6], and the references therein) The connection between the 1 -capacity and the isoperimetric profile is given by:

$$
\inf _{a \leq t \leq b} I(t) \leq \operatorname{cap}_{1}(a, b) \leq \inf _{a \leq t<b} I(t) ; \quad(0<a<b<1) .
$$

Therefore, since we assume the continuity of the isoperimetric profile $I$, we have

$$
\inf _{a \leq t \leq b} I(t)=\operatorname{cap}_{1}(a, b) ; \quad(0<a<b<1) .
$$

(4) Combining (2.3) and (2.4) we get

$$
\frac{1}{\operatorname{cap}_{q}(a, b)} \leq\left(\int_{a}^{b} \frac{d s}{\left(\inf _{s \leq t \leq b} I(t)\right)^{\frac{q}{q-1}}}\right)^{\frac{q-1}{q}} .
$$

Our main result will be formulated using following functions: 
Definition 2. Let $I=I_{(\Omega, d, \mu)}$ be the isoperimetric profile of $(\Omega, d, \mu)$, and let $1 \leq q<\infty$. We let

$$
w_{q}(t)=\left\{\begin{array}{cc}
\left(\frac{1}{t} \int_{0}^{t}\left(\frac{s}{I(s)}\right)^{\frac{q}{q-1}} d s\right)^{\frac{1-q}{q}} & \text { if } q>1 \\
\inf _{0<s<t} \frac{I(s)}{s} & \text { if } q=1 .
\end{array}\right.
$$

Remark 4. Notice that

$$
w_{1}(t) \leq w_{q_{1}}(t) \leq w_{q_{2}}(t) \quad\left(q_{1} \leq q_{2}\right) .
$$

Moreover, if $I(t) / t$ is decreasing, then

$$
\frac{I(t)}{t}=w_{1}(t)
$$

2.2. Symmetrization inequalities under weak assumptions on the isoperimetric profiles.

Theorem 1. Let $(\Omega, d, \mu)$ be a metric probability space that satisfies Conditions 1' and 2 , and let $1 \leq q<\infty$. Then for $f \in \operatorname{Lip}(\Omega) \cap L^{1}(\Omega)$, and for all $t \in(0,1)$, we have

$$
\int_{0}^{t}\left[\left(\left(-f_{\mu}^{*}\right)^{\prime}(\cdot) I(\cdot)\right)^{*}(s)\right]^{q} d s \leq \int_{0}^{t}\left(|\nabla f|_{\mu}^{*}\right)^{q}(s) d s
$$

$$
\left(f_{\mu}^{* *}(t)-f_{\mu}^{*}(t)\right) w_{q}(t) \leq\left(\frac{1}{t} \int_{0}^{t}\left(|\nabla f|_{\mu}^{*}\right)^{q}(s) d s\right)^{1 / q} .
$$

Remark 5. Since $w_{1}(t)=\inf _{0<s<t} \frac{I(s)}{s} \leq I(t)$, it follows readily that, for $q=1$, the inequality (2.9) is weaker than (1.12). On the other hand, (1.12) was proved in 19 under the stronger assumption that $I(t)$ is concave. Now, if $I(t)$ is concave then $\frac{I(t)}{t}$ is decreasing; therefore we have that $w_{1}(t)=I(t)$ (cf. 2.7)) and consequently (2.9) coincides with (1.12).

Remark 6. We do not consider here the corresponding problem of characterizing (2.9) (resp. (2.8)) for $q>1$ in terms of isocapacitary inequalities.

Proof. Since $f \in \operatorname{Lip}(\Omega)$ implies that $|f| \in \operatorname{Lip}(\Omega)$, and, moreover,

$$
|\nabla f(x)| \geq|\nabla| f|(x)|,
$$

we can assume without loss of generality that $f \geq 0$.

Let us start by proving that $f_{\mu}^{*}$ locally absolutely continuous. The proof here follows very closely the one given in $[19$ under the assumption that Condition 1 above holds. Therefore, we will only indicate in detail the changes that are required. Let $0<t_{1}<t_{2}<\infty$, and define

$$
f_{t_{1}}^{t_{2}}(x)= \begin{cases}t_{2}-t_{1} & \text { if } f(x) \geq t_{2} \\ f(x)-t_{1} & \text { if } t_{1}<f(x)<t_{2} \\ 0 & \text { if } f(x) \leq t_{1}\end{cases}
$$

and let 


$$
N\left[f_{t_{1}}^{t_{2}}(x)\right]=\frac{f_{t_{1}}^{t_{2}}(x)}{t_{2}-t_{1}}
$$

It follows that

$$
N\left[f_{t_{1}}^{t_{2}}(x)\right] \text { is } \begin{cases}=1 & \text { if } f(x) \geq t_{2}, \\ <1 & \text { if } t_{1}<f(x)<t_{2} \\ =0 & \text { if } f(x) \leq t_{1} .\end{cases}
$$

By Condition 2,

$$
\left|\nabla N\left[f_{t_{1}}^{t_{2}}(x)\right]\right|=\frac{1}{t_{2}-t_{1}}|\nabla f| \chi_{\left\{t_{1}<|f|<t_{2}\right\}} \mu-\text { a.e, }
$$

and we have

$$
\begin{aligned}
\operatorname{cap}_{1}\left(\mu\left\{|f(x)| \geq t_{2}\right\}, \mu\left\{|f(x)|>t_{1}\right\}\right) & \leq \operatorname{Cap}_{1}\left(\left\{|f(x)| \geq t_{2}\right\}, \quad\left\{|f(x)|>t_{1}\right\}\right) \\
& \leq \int_{\Omega}\left|\nabla N\left[f_{t_{1}}^{t_{2}}(x)\right]\right| d \mu(x) \\
& =\frac{1}{\left(t_{2}-t_{1}\right)} \int_{\left\{t_{1}<f<t_{2}\right\}}|\nabla f(x)| d \mu(x) .
\end{aligned}
$$

Let $0<a<b, t_{1}=f_{\mu}^{*}(b), t_{2}=f_{\mu}^{*}(a)$, then (2.11) yields

$$
\begin{aligned}
& \operatorname{cap}_{1}\left(\mu\left\{|f(x)| \geq f_{\mu}^{*}(a)\right\}, \mu\left\{|f(x)|>f_{\mu}^{*}(b)\right\}\right)\left[f_{\mu}^{*}(a)-f_{\mu}^{*}(b)\right] \\
& \leq \int_{\left\{f_{\mu}^{*}(b)<|f|<f_{\mu}^{*}(a)\right\}}|\nabla f(x)| d \mu(x) .
\end{aligned}
$$

Since

$$
a \leq \mu\left\{|f(x)| \geq f_{\mu}^{*}(a)\right\} \text { and } \mu\left\{|f(x)|>f_{\mu}^{*}(b)\right\}<b,
$$

and $\operatorname{cap}_{q}(.,$.$) is increasing in the first variable and decreasing in the second, we see$ that

$$
\operatorname{cap}_{1}(a, b)\left[f_{\mu}^{*}(a)-f_{\mu}^{*}(b)\right] \leq \int_{\left\{f_{\mu}^{*}(b)<|f|<f_{\mu}^{*}(a)\right\}}|\nabla f(x)| d \mu(x) .
$$

Let us see that $f_{\mu}^{*}$ is locally absolutely continuous. Let us consider an interval $[a, b], 0<a<b<1$. Let $\left\{\left(a_{k}, b_{k}\right)\right\}_{k=1}^{r}$ be any finite family of non-overlapping sub-intervals of $[a, b]$ such that $\sum_{k=1}^{r}\left(b_{k}-a_{k}\right) \leq \delta$. We have

$$
\begin{aligned}
\mu\left\{\cup_{k=1}^{r}\left\{f_{\mu}^{*}\left(b_{k}\right)<|f|<f_{\mu}^{*}\left(a_{k}\right)\right\}\right\} & =\sum_{k=1}^{r} \mu\left\{f_{\mu}^{*}\left(b_{k}\right)<|f|<f_{\mu}^{*}\left(a_{k}\right)\right\} \\
& \leq \sum_{k=1}^{r}\left(b_{k}-a_{k}\right) \leq \delta
\end{aligned}
$$


On the other hand, by (2.12), we have

$$
\begin{aligned}
\sum_{k=1}^{r}\left(f_{\mu}^{*}\left(a_{k}\right)-f_{\mu}^{*}\left(b_{k}\right)\right) \operatorname{cap}_{1}\left(a_{k}, b_{k}\right) & \leq \sum_{k=1}^{r} \int_{\left\{f_{\mu}^{*}\left(b_{k}\right)<|f|<f_{\mu}^{*}\left(a_{k}\right)\right\}}|\nabla| f|(x)| d \mu(x) \\
& =\int_{\cup_{k=1}^{r}\left\{f_{\mu}^{*}\left(b_{k}\right)<|f|<f_{\mu}^{*}\left(a_{k}\right)\right\}}|\nabla| f|(x)| d \mu(x) \\
& \leq\left.\int_{0}^{\delta}|\nabla| f\right|_{\mu} ^{*}(t) d t \\
& \leq \int_{0}^{\delta}|\nabla f|_{\mu}^{*}(t) d t .
\end{aligned}
$$

We also observe that

$$
\operatorname{cap}_{1}\left(a_{k}, b_{k}\right) \geq \operatorname{cap}_{1}\left(a, b_{k}\right)=\operatorname{cap}_{1}\left(1-b_{k}, 1-a\right) \geq \operatorname{cap}_{1}(1-b, 1-a) .
$$

Thus, combining our estimates we see that

$$
\operatorname{cap}_{1}(1-b, 1-a) \sum_{k=1}^{r}\left(f_{\mu}^{*}\left(a_{k}\right)-f_{\mu}^{*}\left(b_{k}\right)\right) \leq \int_{0}^{\delta}|\nabla f|_{\mu}^{*}(t) d t .
$$

The local absolute continuity of $f_{\mu}^{*}$ follows.

In the course of the proof of this theorem we shall also need to know the local absolute continuity of the function $\Psi(t)=\int_{\left\{|f|>f_{\mu}^{*}(t)\right\}}|\nabla f(x)|^{q} d \mu(x)$, under the assumption that $|\nabla f(x)|^{q} \in L^{1}(\Omega)$. This fact can be easily seen with essentially with the same argument we have just provided. Indeed, fix once again an interval $[a, b] \subset$ $(0,1)$, and consider any finite family of non-overlapping sub-intervals $\left\{\left(a_{k}, b_{k}\right)\right\}_{k=1}^{r}$ of $[a, b]$ such that $\sum_{k=1}^{r}\left(b_{k}-a_{k}\right) \leq \delta$. We can then estimate as before

$$
\begin{aligned}
\sum_{k=1}^{r}\left|\Psi\left(b_{k}\right)-\Psi\left(a_{k}\right)\right| & \leq \sum_{k=1}^{r} \int_{\left\{f_{\mu}^{*}\left(b_{k}\right)<|f|<f_{\mu}^{*}\left(a_{k}\right)\right\}}|\nabla f(x)|^{q} d \mu(x) \\
& \leq \int_{0}^{\delta}|\nabla f|_{\mu}^{* q}(t) d t,
\end{aligned}
$$

and the local absolute continuity of $\Psi$ follows.

We now prove (2.8).

Case $q>1$. Let $0<h<t<1$. The same argument that shows (2.12) yields with $a=t-h, b=t$.

$$
\operatorname{cap}_{q}(t-h, t)\left[f_{\mu}^{*}(t-h)-f_{\mu}^{*}(t)\right] \leq\left(\int_{\left\{f_{\mu}^{*}(t)<|f|<f_{\mu}^{*}(t-h)\right\}}|\nabla f(x)|^{q} d \mu(x)\right)^{1 / q} .
$$

Combining with (2.5) we obtain,

$$
\left[\frac{f_{\mu}^{*}(t-h)-f_{\mu}^{*}(t)}{h}\right]\left(\frac{1}{h} \int_{t-h}^{t} \frac{d s}{\left(\inf _{s \leq z \leq t} I(z)\right)^{\frac{q}{q-1}}}\right)^{\frac{1-q}{q}} \leq\left(\frac{1}{h} \int_{\left\{f_{\mu}^{*}(t)<|f|<f_{\mu}^{*}(t-h)\right\}}|\nabla f(x)|^{q} d \mu(x)\right)^{1 / q} .
$$

Letting $h \rightarrow 0$ we find

$$
\left(-f_{\mu}^{*}\right)^{\prime}(t) I(t) \leq\left(\frac{d}{d t} \int_{\left\{|f|>f_{\mu}^{*}(t)\right\}}|\nabla f(x)|^{q} d \mu(x)\right)^{1 / q} .
$$


Consider a finite family of intervals $\left(a_{i}, b_{i}\right), i=1, \ldots, m$, with $0<a_{1}<b_{1} \leq a_{2}<$ $b_{2} \leq \cdots \leq a_{m}<b_{m}<1$. The previous inequality then yields

$$
\begin{aligned}
\int_{\cup_{1 \leq i \leq m}\left(a_{i}, b_{i}\right)}\left(\left(-f_{\mu}^{*}\right)^{\prime}(s) I(s)\right)^{q} d s & \leq \int_{\cup_{1 \leq i \leq m}\left(a_{i}, b_{i}\right)}\left(\frac{d}{d s} \int_{\left\{|f|>f_{\mu}^{*}(s)\right\}}|\nabla f(x)|^{q} d \mu(x)\right) d s \\
& =\sum_{i=1}^{m} \int_{\left\{f_{\mu}^{*}\left(b_{i}\right)<|f| \leq f_{\mu}^{*}\left(a_{i}\right)\right\}}|\nabla f(x)|^{q} d \mu(x) \\
& =\sum_{i=1}^{m} \int_{\left\{f_{\mu}^{*}\left(b_{i}\right)<|f|<f_{\mu}^{*}\left(a_{i}\right)\right\}}|\nabla f(x)|^{q} d \mu(x) \quad \text { (by Condition 2) } \\
& =\int_{\cup_{1 \leq i \leq m}\left\{f_{\mu}^{*}\left(b_{i}\right)<|f|<f_{\mu}^{*}\left(a_{i}\right)\right\}}|\nabla f(x)|^{q} d \mu(x) \\
& \leq \int_{0}^{\sum_{i=1}^{m}\left(b_{i}-a_{i}\right)}\left(|\nabla f|_{\mu}^{*}(s)\right)^{q} d s .
\end{aligned}
$$

Now by a routine limiting process it follows that for any measurable set $E \subset(0,1)$ we have

$$
\int_{E}\left(\left(-f_{\mu}^{*}\right)^{\prime}(s) I(s)\right)^{q} d s \leq \int_{0}^{|E|}\left(|\nabla f|_{\mu}^{*}(s)\right)^{q} d s .
$$

Consequently (2.8) follows from (2.1).

Case $q=1$. Using the same procedure we arrive at

$$
\lim _{h \rightarrow 0} \operatorname{cap}_{1}(t-h, t) \frac{\left[f_{\mu}^{*}(t-h)-f_{\mu}^{*}(t)\right]}{h} \leq \lim _{h \rightarrow 0} \frac{1}{h}\left(\int_{\left\{f_{\mu}^{*}(t)<|f|<f_{\mu}^{*}(t-h)\right\}}|\nabla f(x)| d \mu(x)\right)
$$

which combined with

$$
\operatorname{cap}_{1}(t-h, t) \geq \inf _{t-h \leq z \leq t} I(z)
$$

yields

$$
\left(-f_{\mu}^{*}\right)^{\prime}(t) I(t) \leq \frac{d}{d t} \int_{\left\{|f|>f_{\mu}^{*}(t)\right\}}|\nabla f(x)| d \mu(x),
$$

as desired.

Finally to prove (2.9) we write

$$
f_{\mu}^{*}(s)-f_{\mu}^{*}(t)=\int_{s}^{t}\left(-f_{\mu}^{*}\right)^{\prime}(x) d x
$$

Since $f \in \operatorname{Lip}(\Omega) \cap L^{1}(\Omega), f_{\mu}^{* *}(t)$ is finite for all $0<t \leq 1$. Consequently, by (2.13) and Fubini's theorem, we get

$$
\begin{aligned}
f_{\mu}^{* *}(t)-f_{\mu}^{*}(t) & =\frac{1}{t} \int_{0}^{t}\left(f_{\mu}^{*}(s)-f_{\mu}^{*}(t)\right) d s=\frac{1}{t} \int_{0}^{t}\left(\int_{s}^{t}\left(-f_{\mu}^{*}\right)^{\prime}(x) d x\right) d s \\
& =\frac{1}{t} \int_{0}^{t} s\left(-f_{\mu}^{*}\right)^{\prime}(s) d s .
\end{aligned}
$$


By Hölder's inequality and (2.8),

$$
\begin{aligned}
\int_{0}^{t} s\left(-f_{\mu}^{*}\right)^{\prime}(s) d s & \leq\left(\int_{0}^{t}\left(\left(-f_{\mu}^{*}\right)^{\prime}(s) I(s)\right)^{q} d s\right)^{1 / q} \frac{1}{w_{q}(t)} \\
& \leq\left(\int_{0}^{t}\left[\left(\left(-f_{\mu}^{*}\right)^{\prime}(\cdot) I(\cdot)\right)^{*}(s)\right]^{q} d s\right)^{1 / q} \frac{1}{w_{q}(t)} \\
& \leq\left(\int_{0}^{t}\left(|\nabla f|_{\mu}^{*}\right)^{q}(s) d s\right)^{1 / q} \frac{1}{w_{q}(t)}
\end{aligned}
$$

and (2.9) follows.

\subsection{A version of Theorem 1 without assuming Condition 2.}

Theorem 2. Let $(\Omega, d, \mu)$ be a metric probability space satisfying Condition 1', and let $1 \leq q<\infty$. Then for $f \in \operatorname{Lip}(\Omega) \cap L^{1}(\Omega)$, we have

$$
\left(f_{\mu}^{* *}(t)-f_{\mu}^{*}(t)\right) w_{q}(t) \leq\left(\frac{1}{t} \int_{0}^{t}\left(|\nabla f|_{\mu}^{*}\right)^{q}(s) d s\right)^{1 / q}, \text { for } t \in(0,1) .
$$

Proof. We rely heavily on an argument by Emanuel Milman [26, Remark 3.3] adapted to our setting. Let $\Psi$ be the class of positive Lipschitz functions defined on $\Omega$ that, moreover, satisfy

1) $0 \leq \Phi \leq 1$.

2) For every $0 \leq t \leq 1$,

$$
\int_{\{\Phi=t\}}|\nabla \Phi|^{q} d \mu=0
$$

Given $\Phi \in \Psi$, the truncation $N\left[\Phi_{t_{1}}^{t_{2}}\right]$ (cf. (2.10) above), satisfies

$$
\left(t_{2}-t_{1}\right)\left|\nabla\left(N\left[\Psi_{t_{1}}^{t_{2}}(x)\right]\right)\right| \leq|\nabla \Phi(x)| \text { for all } x \in \Omega .
$$

Moreover, since $N\left[\Psi_{t_{1}}^{t_{2}}(x)\right]$ is constant on the open sets $\left\{\Phi>t_{2}\right\}$ and $\left\{\Phi<t_{1}\right\}$, we have $\left|\nabla\left(N\left[\Phi_{t_{1}}^{t_{2}}\right]\right)\right|=0$ on these sets, and

$$
\begin{aligned}
\int_{\Omega}\left|\nabla\left(N\left[\Phi_{t_{1}}^{t_{2}}(x)\right]\right)\right| d \mu(x) & =\frac{1}{\left(t_{2}-t_{1}\right)} \int_{\left\{t_{1} \leq f \leq t_{2}\right\}}|\nabla \Phi(x)| d \mu(x) \\
& \left.=\frac{1}{\left(t_{2}-t_{1}\right)} \int_{\left\{t_{1}<f<t_{2}\right\}}|\nabla \Phi(x)| d \mu(x) \quad \text { by (2.15) }\right) .
\end{aligned}
$$

Proceeding as in the proof of Theorem 1, we obtain

$$
\left(\Phi_{\mu}^{* *}(t)-\Phi_{\mu}^{*}(t)\right) w_{q}(t) \leq\left(\frac{1}{t} \int_{0}^{t}\left(|\nabla \Phi|_{\mu}^{*}\right)^{q}(s) d s\right)^{1 / q} .
$$

We shall now consider two cases:

Case 1: Suppose that $f \in \operatorname{Lip}(\Omega), f \geq 0$ and $f$ is bounded. Then, without loss of generality, we may assume (dividing by a constant if it were necessary) that $\|f\|_{\infty} \leq 1$. It follows from [26. Remark 3.3] that given $\varepsilon>0$ there exists $f_{\varepsilon} \in \Psi$ such that

$$
\left\|\left|\nabla f_{\varepsilon}\right|\right\|_{L^{q}} \leq(1+\varepsilon)\||\nabla f|\|_{L^{q}} .
$$

Moreover, if we denote

$$
\Gamma=\{t \in[0,1]: \mu\{\{f=t\}>0\},
$$


the discrete countable set of atoms of $f$ under $\mu$, then

$$
\int_{\Gamma}\left|\nabla f_{\varepsilon}\right|^{q} d \mu(x)=0 .
$$

Furthermore, let us write $\Gamma=\left\{\gamma_{i}\right\}_{i=0,1,2 \ldots}$, with $\gamma_{i}<\gamma_{i+1}$, and $G_{i}=\left\{x: \gamma_{i}<f(x)<\gamma_{i+1}\right\}$, $i=0,1$.. A perusal of the construction used by E. Milman, shows that on each $G_{i}, i=0,1 . .$, we have

$$
\left|\nabla f_{\varepsilon}(x)\right| \leq(1+\varepsilon)|\nabla f(x)| .
$$

Moreover, if we let $\varepsilon=1 / n$, then

$$
f_{n} \underset{n \rightarrow \infty}{\rightarrow} f \text { in } L^{1} .
$$

Since $f_{n} \in \Psi$, the truncation argument of Theorem 1 works, and we find

$$
\left(\left(f_{n}\right)_{\mu}^{* *}(t)-\left(f_{n}\right)_{\mu}^{*}(t)\right) \leq\left(\frac{1}{t} \int_{0}^{t}\left(\frac{s}{I(s)}\right)^{\frac{q}{q-1}} d s\right)^{\frac{q-1}{q}}\left(\frac{1}{t} \int_{0}^{t}\left(\left|\nabla f_{n}\right|_{\mu}^{*}\right)^{q}(s) d s\right)^{1 / q} .
$$

Therefore, for each $n \in N$ and for any Borel set $E \subset \Omega$ with $\mu(E) \leq t$, we have

$$
\begin{aligned}
\int_{E}\left|\nabla f_{n}\right|^{q} d \mu(x) & =\int_{\Gamma \cap E}\left|\nabla f_{n}\right|^{q} d \mu(x)+\int_{E \backslash \Gamma}\left|\nabla f_{n}\right|^{q} d \mu(x) \\
& =\int_{E \backslash \Gamma}\left|\nabla f_{n}\right|^{q} d \mu(x) \quad(\text { by } 2.16) \\
& =\sum_{i} \int_{G_{i}}\left|\nabla f_{n}\right|^{q} d \mu(x) \\
& \left.\leq\left(1+\frac{1}{n}\right)^{q} \int_{E \backslash \Gamma}|\nabla f|^{q} d \mu(x) \quad \text { (by (2.17) }\right) \\
& \leq\left(1+\frac{1}{n}\right)^{q} \int_{E}|\nabla f|^{q} d \mu(x) .
\end{aligned}
$$

Consequently, by (2.1), we obtain

$$
\begin{aligned}
\int_{0}^{t}\left(\left|\nabla f_{n}\right|_{\mu}^{*}\right)^{q}(s) d s & =\sup _{\mu(E) \leq t} \int_{E}\left|\nabla f_{n}\right|^{q} d \mu(x) \\
& \leq\left(1+\frac{1}{n}\right)^{q} \sup _{\mu(E) \leq t} \int_{E}|\nabla f|^{q} d \mu(x) \\
& =\left(1+\frac{1}{n}\right)^{q} \int_{0}^{t}\left(|\nabla f|_{\mu}^{*}\right)^{q}(s) d s .
\end{aligned}
$$

On the other hand from (2.18) we get (cf. [12, Lemma 2.1]):

$$
\begin{aligned}
& \left(f_{n}\right)_{\mu}^{* *}(t) \rightarrow f_{\mu}^{* *}(t), \text { uniformly for } t \in[0,1], \text { and } \\
& \left(f_{n}\right)_{\mu}^{*}(t) \rightarrow f_{\mu}^{*}(t) \text { at all points of continuity of } f_{\mu}^{*} .
\end{aligned}
$$

Thus, letting $n \rightarrow \infty$ we obtain (2.14).

Case 2: Suppose that $f$ is a positive Lip function. Consider an increasing sequence of positive number $a_{n}$ such that $\lim _{n} a_{n}=\infty$, and such that, moreover, the sets $D_{n}=\left\{x: f(x)=a_{n}\right\}$ have $\mu$-measure 0 , for all $n$. Let

$$
h_{n}=\left\{\begin{array}{cc}
a_{n} & \text { if } f(x) \geq a_{n}, \\
f(x) & \text { if } f(x)<a_{n}
\end{array}\right.
$$


Apply the result obtained in the first part of the proof to each of the $h_{n}^{\prime} s$. We obtain (2.19)

$\left(\left(h_{n}\right)_{\mu}^{* *}(t)-\left(h_{n}\right)_{\mu}^{*}(t)\right) \leq\left(\frac{1}{t} \int_{0}^{t}\left(\frac{s}{I(s)}\right)^{\frac{q}{q-1}} d s\right)^{\frac{q-1}{q}}\left(\frac{1}{t} \int_{0}^{t}\left(\left|\nabla h_{n}\right|_{\mu}^{*}\right)^{q}(s) d s\right)^{1 / q}$.

Since for each $n \in N$ the set $A_{n}=\left\{x: f(x)<a_{n}\right\}$ is open, we have $\left|\nabla h_{n}(x)\right|=$ $|\nabla f(x)|$, a.e. $x \in A_{n}$.

Given a measurable set $E \subset \Omega$, with $\mu(E) \leq t$,

$$
\begin{aligned}
\int_{E}\left|\nabla h_{n}\right|^{q} d \mu & =\int_{E \cap A_{n}}\left|\nabla h_{n}\right|^{q} d \mu+\int_{E \backslash A_{n}}\left|\nabla h_{n}\right|^{q} d \mu \\
& =\int_{\Gamma \cap A_{n}}\left|\nabla h_{n}\right|^{q} d \mu \quad\left(\text { since } \mu\left(D_{n}\right)=0\right) \\
& =\int_{E \cap A_{n}}|\nabla f|^{q} d \mu \\
& \leq \int_{E}|\nabla f|^{q} d \mu .
\end{aligned}
$$

Thus

$$
\int_{0}^{t}\left(\left|\nabla h_{n}\right|_{\mu}^{*}\right)^{q}(s) d s \leq \int_{0}^{t}\left(|\nabla f|_{\mu}^{*}\right)^{q}(s) d s .
$$

To take care of the left hand side of (2.19) we can use again [12, Lemma 2.1] noting that, by monotone convergence,

$$
h_{n} \underset{n \rightarrow \infty}{\rightarrow} f \text { in } L^{1} .
$$

Combining our findings we can conclude the proof of (2.14).

2.4. Poincaré inequalities on r.i. spaces. From now on we will assume that our metric probability spaces $(\Omega, d, \mu)$ satisfy Condition 1 '.

We consider Banach function spaces on $(\Omega, d, \mu)$ with the property if $g \in X$ and $f$ is a $\mu$-measurable function on $\Omega$ such that $f_{\mu}^{*}=g_{\mu}^{*}$, then $f \in X$, and, moreover, $\|f\|_{X}=\|g\|_{X}$. We say that $X=X(\Omega)$ is a rearrangement-invariant (r.i.) spact21. It follows that

$$
L^{\infty}(\Omega) \subset X(\Omega) \subset L^{1}(\Omega),
$$

with continuous embeddings.

An r.i. space $X=X(\Omega)$ can be represented by a r.i. space $\bar{X}=\bar{X}(0,1)$ on the interval $(0,1)$, with Lebesgue measure 13 in the sense that for $f \in X$,

$$
\|f\|_{X}=\left\|f_{\mu}^{*}\right\|_{\bar{X}}
$$

Let us also record here the Hardy-Calderón property

$$
f_{\mu}^{* *} \leq g_{\mu}^{* *} \Rightarrow\|f\|_{X} \leq\|g\|_{X} .
$$

Typical examples of r.i. spaces are the $L^{p}$-spaces, Lorentz spaces and Orlicz spaces.

\footnotetext{
${ }^{12}$ We refer the reader to [5] for a detailed treatment.

${ }^{13} \mathrm{~A}$ characterization of the norm $\|\cdot\|_{\bar{X}}$ is available (see [5] Theorem 4.10 and subsequent remarks])
} 
The Boyd indices, $\bar{\alpha}_{X}, \underline{\alpha}_{X}$, of a r.i. space $X$ (cf. [5] for details) are defined by

$$
\bar{\alpha}_{X}=\inf _{s>1} \frac{\ln h_{X}(s)}{\ln s} \quad \text { and } \quad \underline{\alpha}_{X}=\sup _{s<1} \frac{\ln h_{X}(s)}{\ln s},
$$

where $h_{X}(s)$ denotes the norm of the dilation operator $E_{s}, s>0$, on $\bar{X}$, defined by 14

$$
E_{s} f(t)= \begin{cases}f^{*}\left(\frac{t}{s}\right) & 0<t<s \\ 0 & s<t<1 .\end{cases}
$$

For example, if $X=L^{p}$, then $\bar{\alpha}_{L^{p}}=\underline{\alpha}_{L^{p}}=\frac{1}{p}$. Consider the Hardy operators defined by

$$
P f(t)=\frac{1}{t} \int_{0}^{t} f(s) d s ; \quad Q f(t)=\int_{t}^{\infty} f(s) \frac{d s}{s} .
$$

It is well known that (cf. [5] )

$$
\begin{gathered}
P \text { is bounded on } \bar{X} \Leftrightarrow \bar{\alpha}_{X}<1, \\
Q_{a} \text { is bounded on } \bar{X} \Leftrightarrow \underline{\alpha}_{X}>a .
\end{gathered}
$$

The $q$-convexification $X^{(q)}$ of a r.i. space $X$ are defined by the condition

$$
X^{(q)}=\left\{f:|f|^{q} \in X\right\}, \quad\|f\|_{X^{(q)}}=\left\||f|^{q}\right\|_{X}^{1 / q} .
$$

The $q$-capacitary spaces $L S_{q}(X)$ associated with a r.i. space $X$ are defined by the condition

$$
\|f\|_{L S_{q}(X)}:=\left\|\left(f_{\mu}^{* *}(t)-f_{\mu}^{*}(t)\right) w_{q}(t)\right\|_{\bar{X}}<\infty .
$$

It follows from (2.6) that these functionals increase with the parameter $q$.

The $q$-capacitary Hardy operator $Q_{w_{q}}$ is defined on positive measurable positive functions on $(0,1)$ by

$$
Q_{w_{q}} f(t)=\int_{t}^{1} f(s) \frac{d s}{s w_{q}(s)} .
$$

2.4.1. Poincaré inequalities and the capacitary Hardy operator $Q_{w_{q}}$.

Theorem 3. Let $X(\Omega), Y(\Omega)$ be r.i. spaces such that $\bar{\alpha}_{X}<1$. Let $q \geq 1$, and suppose that there exists a constant $c=c(q)$ such that for every positive function $f \in \bar{X}^{(q)}$, with supp $\subset \subset(0,1 / 2)$, we have

$$
\left\|Q_{w_{q}} f\right\|_{\bar{Y}^{(q)}} \leq c\|f\|_{\bar{X}^{(q)}} .
$$

Then there exists a constant $C=C\left(c,\|P\|_{\bar{X} \rightarrow \bar{X}}\right)$ such that for all $g \in \operatorname{Lip}(\Omega) \cap$ $L^{1}(\Omega)$,

$$
\left\|g-\int_{\Omega} g d \mu\right\|_{Y^{(q)}} \leq C\left(\||\nabla g|\|_{X^{(q)}}+\left\|g-\int_{\Omega} g d \mu\right\|_{L^{1}}\right)
$$

${ }^{14}$ The operator $E_{s}$ is bounded on $\bar{X}$ for every r.i. space $X(\Omega)$ and for every $s>0$. Moreover,

$$
h_{X}(s) \leq \max \{1, s\} .
$$


Moreover,

$$
\begin{aligned}
\left\|g-\int_{\Omega} g d \mu\right\|_{Y^{(q)}} & \leq C\left\|g-\int_{\Omega} g d \mu\right\|_{L S_{q}\left(X^{(q)}\right)} \\
& \leq C\left(\||\nabla g|\|_{X^{(q)}}+\left\|g-\int_{\Omega} g d \mu\right\|_{L^{1}}\right) .
\end{aligned}
$$

Proof. Since $g \in \operatorname{Lip}(\Omega) \cap L^{1}(\Omega), g_{\mu}^{* *}(t)$ is finite for all $0<t \leq 1$, thus

$$
\begin{aligned}
g_{\mu}^{*}(t)^{q} & \leq g_{\mu}^{* *}(t)^{q}=\left(\int_{t}^{1 / 2}\left(-g_{\mu}^{* *}\right)^{\prime}(s) d s+g_{\mu}^{* *}(1 / 2)\right)^{q} \\
& =\left(\int_{t}^{1 / 2}\left(g_{\mu}^{* *}(s)-g_{\mu}^{*}(s)\right) \frac{d s}{s}+g_{\mu}^{* *}(1 / 2)\right)^{q} \\
& =\left(\int_{t}^{1 / 2}\left(g_{\mu}^{* *}(s)-g_{\mu}^{*}(s)\right) w_{q}(s) \frac{d s}{w_{q}(s) s}+g_{\mu}^{* *}(1 / 2)\right)^{q} .
\end{aligned}
$$

Consequently,

$$
\begin{aligned}
\|g\|_{Y^{(q)}} & =\left\|\left(g_{\mu}^{*}\right)^{q}\right\|_{\bar{Y}}^{1 / q} \\
& \leq\left\|\left(\int_{t}^{1 / 2}\left(g_{\mu}^{* *}(s)-g_{\mu}^{*}(s)\right) w_{q}(s) \frac{d s}{w_{q}(s) s}+g_{\mu}^{* *}(1 / 2)\right)^{q}\right\|_{\bar{Y}}^{1 / q} \\
& =\left\|\int_{t}^{1 / 2}\left(g_{\mu}^{* *}(s)-g_{\mu}^{*}(s)\right) w(s) \frac{d s}{w_{q}(s) s}+g_{\mu}^{* *}(1 / 2)\right\|_{\bar{Y}^{(q)}} \\
& \preceq\left\|\int_{t}^{1 / 2}\left(g_{\mu}^{* *}(s)-g_{\mu}^{*}(s)\right) w_{q}(s) \frac{d s}{w_{q}(s) s}\right\|_{\bar{Y}^{(q)}}+\|g\|_{L^{1}} \\
& \preceq\left\|\left(g_{\mu}^{* *}(s)-g_{\mu}^{*}(s)\right) w_{q}(s)\right\|_{\bar{X}^{(q)}}+\|g\|_{L^{1}} \\
& =\left\|\left[\left(g_{\mu}^{* *}(s)-g_{\mu}^{*}(s)\right) w_{q}(s)\right]^{q}\right\|_{\bar{X}}^{1 / q}+\|g\|_{L^{1}} \\
& \preceq\left\|\frac{1}{t} \int_{0}^{t}\left(|\nabla g|_{\mu}^{*}\right)^{q}(s) d s\right\|_{\bar{X}}^{1 / q}+\|g\|_{L^{1}} \quad(\text { by }(2.9)) \\
& \preceq\left\|\left(|\nabla g|_{\mu}^{*}\right)^{q}\right\|_{\bar{X}}^{1 / q}+\|g\|_{L^{1}} \quad\left(\text { since } \bar{\alpha}_{X}<1\right) \\
& =\left\||\nabla g|_{\mu}^{*}\right\|_{\bar{X}^{(q)}}+\|g\|_{L^{1}} \\
& =\||\nabla g|\|_{X^{(q)}}+\|g\|_{L^{1}} .
\end{aligned}
$$

Therefore,

$$
\left\|g-\int_{\Omega} g d \mu\right\|_{Y^{(q)}} \preceq\||\nabla g|\|_{X^{(q)}}+\left\|g-\int_{\Omega} g d \mu\right\|_{L^{1}} .
$$

Notice that (2.26) is implicit in the proof.

Remark 7. If Cheeger's inequality holds for $(\Omega, d, \mu)$, i.e. if there exists $C_{e}$ such that

$$
\left\|g-\int_{\Omega} g d \mu\right\|_{L^{1}} \leq C_{e}\||\nabla g|\|_{L^{1}},
$$


then the extra $L^{1}$ term that appears in (2.25) and (2.26) can be left out. Indeed, combining Cheeger's inequality with (2.20) yields

$$
\left\|g-\int_{\Omega} g d \mu\right\|_{L^{1}} \leq C_{e}\||\nabla g|\|_{L^{1}} \leq C_{e} \bar{c}\||\nabla g|\|_{X^{(q)}},
$$

where $\bar{c}$ denotes the embedding constant of $X^{(q)} \subset L^{1}$ (cf. (2.20)).

Remark 8. For $q=1$, the condition 2.24) reads: there exists a constant $C$ such that for every positive function $f \in \bar{X}$ with supp $\subset \subset(0,1 / 2)$, we have

$$
\left\|\int_{t}^{1} f(s) \frac{d s}{s\left(\inf _{0<z<t} \frac{I(z)}{z}\right)}\right\|_{\bar{Y}} \leq C\|f\|_{\bar{X}} .
$$

2.4.2. Poincaré inequalities: a limiting case. The limiting case where $\bar{\alpha}_{X}=1$ is not covered by Theorem 3 . For example, if $X=L^{1}$ then the condition (2.26) reads as

$$
\left\|g-\int_{\Omega} g d \mu\right\|_{L^{q}} \preceq\left\|g-\int_{\Omega} g d \mu\right\|_{L S\left(L^{q}\right)} \preceq\||\nabla g|\|_{L^{q}},
$$

but unfortunately we cannot apply the theorem since $\bar{\alpha}_{L^{1}}=1$.

In this section we formulate conditions for the validity of Poincaré inequalities in terms of $\nu(s)=\operatorname{cap}_{1}(s, 1 / 2) / s$. Let $q \in[1, \infty)$, then we say that $\nu$ is a $q$-Muckenhoupt weight iff there exists a constant $c>0$ such that

$$
\|P f\|_{L^{q}(\nu)} \leq c\|f\|_{L^{q}(\nu)} .
$$

Using the usual description of Muckenhoupt weights (cf. [27, [28]) we see that $\nu(s)=\operatorname{cap}_{1}(s, 1 / 2) / s$ is a $q$-Muckenhoupt weight iff there exists a constant $c>0$ such that for all $0<t<1 / 2$,

$$
\left\{\begin{array}{cc}
\left(\int_{t}^{1 / 2}\left(\frac{c a p_{1}(s, 1 / 2)}{s}\right)^{q} \frac{d s}{s^{q}}\right)^{1 / q}\left(\int_{0}^{t}\left(\frac{s}{\operatorname{cap}_{1}(s, 1 / 2)}\right)^{\frac{q}{q-1}} d s\right)^{\frac{q-1}{q}} \leq c & \text { if } 1<q, \\
\int_{t}^{1 / 2} \frac{c a p_{1}(s, 1 / 2)}{s} \frac{d s}{s} \leq c \frac{c a p_{1}(t, 1 / 2)}{t} & \text { if } q=1 .
\end{array}\right.
$$

We now show that if $\nu$ is $q$-Muckenhoupt weight then Poincaré inequalities can be described in terms of the Hardy isoperimetric operator

$$
Q_{c a p_{1}} f(t)=\int_{t}^{1 / 2} f(s) \frac{d s}{\operatorname{cap}_{1}(s, 1 / 2)} .
$$

Theorem 4. Let $q \geq 1$, and suppose that $\frac{\text { cap }_{1}(t, 1 / 2)}{t}$ is a $q$-Muckenhoupt weight. Then, there exists a constant $c>0$ such that for all $f \in \operatorname{Lip}(\Omega) \cap L^{1}(\Omega), 0<t<1 / 2$, we have

$$
\int_{0}^{t}\left[\left(f_{\mu}^{* *}(s)-f_{\mu}^{*}(s)\right) \frac{\operatorname{cap}_{1}(s, 1 / 2)}{s}\right]^{q} d s \leq c \int_{0}^{t}\left(|\nabla f|_{\mu}^{*}\right)^{q}(s) d s .
$$

In particular, if $X$ is a r.i. space with $\underline{\alpha}_{X}>0$, there exists an absolute constant $C$ (depending on $c$, the $q-M$-Mckenhoupt norm of $\frac{c a p_{1}(t, 1 / 2)}{t}$, and the norms of the Hardy operators $P, Q)$ such that for all $f \in \operatorname{Lip}(\Omega) \cap L^{1}(\Omega)$,

$$
\left\|\left[f_{\mu}^{* *}(t)-f_{\mu}^{*}(t)\right] \frac{\operatorname{cap}_{1}(t, 1 / 2)}{t}\right\|_{\bar{X}^{(q)}} \leq C\left(\||\nabla f|\|_{X^{(q)}}+\left\|f-\int_{\Omega} f d \mu\right\|_{L^{1}}\right) .
$$


Moreover, suppose that there exists $\tilde{C}>0$ such that for every positive function $f \in \bar{X}^{(q)}$, we have

$$
\left\|Q_{\text {cap } 1} f\right\|_{\bar{Y}^{(q)}} \leq \tilde{C}\|f\|_{\bar{X}^{(q)}}
$$

Then, there exist absolute constants $C_{1}, C_{2}$ (that depend on all the previous constants as well as $\tilde{C})$ such that for all $g \in \operatorname{Lip}(\Omega) \cap L^{1}(\Omega)$,

$$
\begin{aligned}
\left\|g-\int_{\Omega} g d \mu\right\|_{Y^{(q)}} & \leq C_{1}\left\|\left[\left(g-\int_{\Omega} g d \mu\right)_{\mu}^{* *}(t)-\left(g-\int_{\Omega} g d \mu\right)_{\mu}^{*}(t)\right] \frac{\operatorname{cap}_{1}(t, 1 / 2)}{t}\right\|_{\bar{X}^{(q)}} \\
& \leq C_{2}\left(\|\nabla g\|_{X^{(q)}}+\left\|g-\int_{\Omega} g d \mu\right\|_{L^{1}}\right) .
\end{aligned}
$$

Proof. Suppose that $0 \leq f \in \operatorname{Lip}(\Omega) \cap L^{1}$ satisfies

(2.30) For every $c \in \mathbb{R}$, we have that $|\nabla f(x)|=0, \mu-$ a.e. on $\{x: f(x)=c\}$.

Then by the proof of Theorem $1, f_{\mu}^{*}$ is locally absolutely continuous, and

$$
f_{\mu}^{* *}(t)-f_{\mu}^{*}(t)=\frac{1}{t} \int_{0}^{t} s\left(-f_{\mu}^{*}\right)^{\prime}(s) d s .
$$

Thus,

$$
\begin{aligned}
\int_{0}^{t}\left[\left(f_{\mu}^{* *}(s)-f_{\mu}^{*}(s)\right) \frac{c a p_{1}(s, 1 / 2)}{s}\right]^{q} d s & =\int_{0}^{t}\left[\left(\frac{1}{s} \int_{0}^{s} z\left(-f_{\mu}^{*}\right)^{\prime}(z) d z\right) \frac{\operatorname{cap}_{1}(s, 1 / 2)}{s}\right]^{q} d s \\
& \leq c \int_{0}^{t}\left[\left(\left(-f_{\mu}^{*}\right)^{\prime}(s)\right) \operatorname{cap}_{1}(s, 1 / 2)\right]^{q} d s \quad \text { (by (2.27) } \\
& =c \int_{0}^{t}\left[\left(\left(-f_{\mu}^{*}\right)^{\prime}(s)\right) \inf _{s \leq z<1 / 2} I(z)\right]^{q} d s \quad \text { (by (2.4) ) } \\
& \leq c \int_{0}^{t}\left[\left(\left(-f_{\mu}^{*}\right)^{\prime}(s)\right) I(s)\right]^{q} d s \\
& \leq c \int_{0}^{t}\left(|\nabla f|_{\mu}^{*}\right)^{q}(s) d s \quad \text { (by (2.8). }
\end{aligned}
$$

Now, using a familiar limiting argument we can avoid the restriction (2.30) and still achieve (2.29).

Applying the operator $Q_{1 / 2} f(s)=\int_{s}^{1 / 2} f(z) \frac{d z}{z}$ to the inequality (2.28), and then combining with the fact that for $0<t<1 / 2$ we have (see [4])

$$
\begin{array}{r}
P\left(Q_{1 / 2} f\right)(s)-2 \int_{0}^{1 / 2} f=Q_{1 / 2}(P f)(s), \\
P f(s)+Q_{1 / 2} f(s)=P\left(Q_{1 / 2} f\right)(s),
\end{array}
$$

we obtain

$$
\begin{aligned}
& \frac{1}{t} \int_{0}^{t} Q_{1 / 2}\left[\left(f_{\mu}^{* *}(\cdot)-f_{\mu}^{*}(\cdot)\right) \frac{c a p(\cdot, 1 / 2)}{(\cdot)}\right]^{q}(s) d s-2 \int_{0}^{1 / 2}\left[\left(f_{\mu}^{* *}(s)-f_{\mu}^{*}(s)\right) \frac{c a p(\cdot, 1 / 2)}{s}\right]^{q} d s \\
& \leq c\left(\frac{1}{t} \int_{0}^{t} Q_{1 / 2}\left(\left(|\nabla f|_{\mu}^{*}\right)^{q}(s)\right) d s-2 \int_{0}^{1 / 2}\left(|\nabla f|_{\mu}^{*}\right)^{q}(s)\right) \\
& \leq \frac{c}{t} \int_{0}^{t} Q_{1 / 2}\left(\left(|\nabla f|_{\mu}^{*}\right)^{q}(s)\right) d s .
\end{aligned}
$$


Moreover, since

$$
\begin{aligned}
\frac{1}{1 / 2} \int_{0}^{1 / 2}\left[\left(f_{\mu}^{* *}(s)-f_{\mu}^{*}(s)\right) \frac{c a p_{1}(s, 1 / 2)}{s}\right]^{q} d s & \leq \frac{1}{t} \int_{0}^{t}\left[\left(f_{\mu}^{* *}(s)-f_{\mu}^{*}(s)\right) \frac{\operatorname{cap}_{1 / 2}(s, 1 / 2)}{s}\right]^{q} d s \\
& \leq \frac{c}{t} \int_{0}^{t}\left(|\nabla f|_{\mu}^{*}\right)^{q}(s) d s \quad(\text { by (2.28) }) \\
& \leq \frac{c}{t} \int_{0}^{t} Q_{1 / 2}\left(\left(|\nabla f|_{\mu}^{*}\right)^{q}(s)\right) d s \quad(\text { by (2.31) }),
\end{aligned}
$$

we obtain

$$
\int_{0}^{t} Q_{1 / 2}\left[\left(f_{\mu}^{* *}(\cdot)-f_{\mu}^{*}(\cdot)\right) \frac{\operatorname{cap}_{1}(\cdot, 1 / 2)}{(\cdot)}\right]^{q}(s) d s \leq 2 c \int_{0}^{t} Q_{1 / 2}\left(\left(|\nabla f|_{\mu}^{*}\right)^{q}\right)(s) d s .
$$

Observe that $Q_{1 / 2} h(s)$ is decreasing. Consequently,

$$
\begin{aligned}
\int_{0}^{t} Q_{1 / 2}\left[\left(f_{\mu}^{* *}(\cdot)-f_{\mu}^{*}(\cdot)\right) \frac{\operatorname{cap}_{1}(\cdot, 1 / 2)}{(\cdot)}\right]^{q}(s) d s & =\int_{0}^{t}\left(Q_{1 / 2}\left[\left(f_{\mu}^{* *}(\cdot)-f_{\mu}^{*}(\cdot)\right) \frac{\operatorname{cap}_{1}(\cdot, 1 / 2)}{(\cdot)}\right]^{q}\right)^{*}(s) d s \\
& \leq 2 c \int_{0}^{t} Q_{1 / 2}\left(\left(|\nabla f|_{\mu}^{*}\right)^{q}\right)(s) d s
\end{aligned}
$$

We may now apply 2.21)

$$
\left\|Q_{1 / 2}\left[\left(f_{\mu}^{* *}(\cdot)-f_{\mu}^{*}(\cdot)\right) \frac{\operatorname{cap}_{1}(\cdot, 1 / 2)}{(\cdot)}\right]^{q}(t)\right\|_{X} \leq C\left\|Q_{1 / 2}\left(\left(|\nabla f|_{\mu}^{*}\right)^{q}\right)(t)\right\|_{X} .
$$

Whence, if $\underline{\alpha}_{X}>0$,

$$
\left\|Q_{1 / 2}\left[\left(f_{\mu}^{* *}(\cdot)-f_{\mu}^{*}(\cdot)\right) \frac{\operatorname{cap}_{1}(\cdot, 1 / 2)}{(\cdot)}\right]^{q}(t)\right\|_{\bar{X}} \leq C\left\||\nabla f|^{q}\right\|_{X} .
$$

Since both, $t \rightarrow t\left(f_{\mu}^{* *}(t)-f_{\mu}^{*}(t)\right)$ and $t \rightarrow \operatorname{cap}_{1}(t, 1 / 2)$ are increasing, we see that for $0<t<1 / 4$

$$
\begin{aligned}
& \int_{t}^{1 / 2}\left[\left(f_{\mu}^{* *}(s)-f_{\mu}^{*}(s)\right) \frac{\operatorname{cap}_{1}(s, 1 / 2)}{s}\right]^{q} \frac{d s}{s} \\
& \geq\left(t\left(f_{\mu}^{* *}(t)-f_{\mu}^{*}(t)\right) \operatorname{cap}_{1}(t, 1 / 2)\right)^{q} \int_{t}^{1 / 2}\left[\frac{1}{s^{2}}\right]^{q} \frac{d s}{s} \\
& \succeq\left(\left(f_{\mu}^{* *}(t)-f_{\mu}^{*}(t)\right) \frac{\operatorname{cap}_{1}(t, 1 / 2)}{t}\right)^{q} .
\end{aligned}
$$

Using the elementary estimation

$$
\|h\|_{\bar{X}} \preceq\left\|h \chi_{(0,1 / 4)}\right\|_{\bar{X}}
$$

we find

$$
\begin{aligned}
\left\|\left(\left[f_{\mu}^{* *}(t)-f_{\mu}^{*}(t)\right] \frac{\operatorname{cap}_{1}(t, 1 / 2)}{t}\right)^{q}\right\|_{\bar{X}} & \preceq\left\|\left(\left[f_{\mu}^{* *}(t)-f_{\mu}^{*}(t)\right] \frac{\operatorname{cap}_{1}(t, 1 / 2)}{t}\right)^{q} \chi_{(0,1 / 4)}(t)\right\|_{\bar{X}} \\
& \preceq\left\|Q_{1 / 2}\left[\left(f_{\mu}^{* *}(\cdot)-f_{\mu}^{*}(\cdot)\right) \frac{\operatorname{cap}(\cdot, 1 / 2)}{(\cdot)}\right]^{q}(t) \chi_{(0,1 / 4)}(t)\right\|_{\bar{X}} \\
& \preceq\left\|\left(|\nabla f|_{\mu}^{*}\right)^{q}\right\|_{X} .
\end{aligned}
$$


Finally, to see (2.30), we proceed as in theorem 3 .

$$
f_{\mu}^{*}(t)^{q} \leq f_{\mu}^{* *}(t)^{q}=\left(\int_{t}^{1 / 2}\left[f_{\mu}^{* *}(t)-f_{\mu}^{*}(t)\right] \frac{\operatorname{cap}_{1}(s, 1 / 2)}{s} \frac{d s}{\operatorname{cap}_{1}(s, 1 / 2)}+f_{\mu}^{* *}(1 / 2)\right)^{q}
$$

Consequently,

$$
\begin{aligned}
\left\|f_{\mu}^{*}\right\|_{Y^{(q)}} & =\left\|\left(f_{\mu}^{* *}\right)^{q}\right\|_{\bar{Y}}^{1 / q} \\
& \leq\left\|\left(\int_{t}^{1 / 2}\left[f_{\mu}^{* *}(t)-f_{\mu}^{*}(t)\right] \frac{\operatorname{cap}_{1}(s, 1 / 2)}{s} \frac{d s}{\operatorname{cap}_{1}(s, 1 / 2)}+f_{\mu}^{* *}(1 / 2)\right)^{q}\right\|_{\bar{Y}}^{1 / q} \\
& \preceq\left\|\left(f_{\mu}^{* *}(s)-f_{\mu}^{*}(s)\right) \frac{\operatorname{cap}_{1}(s, 1 / 2)}{s}\right\|_{\bar{X}^{(q)}}+\|f\|_{L^{1}} \\
& \left.\preceq\left\|\left(|\nabla f|_{\mu}^{*}\right)^{q}\right\|_{\bar{X}}^{1 / q}+\|f\|_{L^{1}} \quad \text { (by (2.29) }\right) .
\end{aligned}
$$

Remark 9. Observe that for $0<t<1 / 2$,

$$
\frac{\operatorname{cap}_{1}(t, 1 / 2)}{t}=\frac{\inf _{t \leq z<1 / 2} I(z)}{t}=\inf _{0<s<t} \frac{\inf _{t \leq s<1 / 2} I(s)}{s} \leq \inf _{0<s<t} \frac{I(s)}{s}=w_{1}(t) .
$$

Thus

$$
\left\|\left[g_{\mu}^{* *}(t)-g_{\mu}^{*}(t)\right] \frac{\operatorname{cap}_{1}(t, 1 / 2)}{t}\right\|_{\bar{X}^{(q)}} \preceq\left\|\left[g_{\mu}^{* *}(t)-g_{\mu}^{*}(t)\right] w_{1}(t)\right\|_{\bar{X}^{(q)}} .
$$

Remark 10. As in Theorem 3, the extra $L^{1}$-terms appearing in (2.29) and (2.30) can be omitted if Cheeger's inequality holds. Notice that Cheeger's inequality is equivalent to (cf. 25] and the references therein)

$$
\operatorname{cap}_{1}(t, 1 / 2) \geq \operatorname{ct} \quad(0<t \leq 1 / 2)
$$

which in turn is equivalent to (cf. [19])

$$
\left\|Q_{\text {cap }_{1}} f\right\|_{L^{1}} \leq C\|f\|_{L^{1}}
$$

for all positive functions $f \in L^{1}$, with supp $f \subset(0,1 / 2)$.

\section{A connection with martingale inequalities via interpolation theory and optimization}

It was shown recently in [10] that using interpolation theory one can relate the rearrangement inequalities for Sobolev functions we have obtained in our work with the extrapolation theory of martingale inequalities of Burkholder and Gundy 8] and Herz [14. There are two key observations underlying these developments: (i) the idea to treat truncations as part of the more general process of decomposing elements. Here the appropriate setting is the real method of interpolation, where decompositions are selected using penalty methods. From this point of view our method is related to the fact that certain optimal splittings in interpolation theory are given by truncations; (ii) the fact that gradients (and other related operations in analysis, e.g. the martingale square functions!) commute, in suitable quantified manners, with respect to these splittings.

We thought it would be worthwhile to present these ideas to this community using a presentation that goes directly to the heart of the matter. Thus we will 
focus our discussion here on Sobolev inequalities and refer the reader to $\mathbf{1 0}$ for complete proofs and other developments. This topic will also be discussed in [20. One reason we were originally interested in placing our results in a larger context is that it may help to suggest a suitable substitute for the truncation method when we deal with higher order differential operators where truncations are obviously inadequate.

We start by placing the truncation method within the larger context of interpolation theory. The basic modern ingredient of *real interpolation* is the study of controlled splittings of elements using "penalty" methods ("Peetre's $K$-functional). The point of departure of this theory are pairs $\vec{X}=\left(X_{0}, X_{1}\right)$ of Banach spaces that are "compatible" in the sense that both spaces $X_{i}, i=0,1$, are continuously embedded in a common Hausdorff topological vector spact15. For such pairs the sum space $\Sigma(\vec{X})=X_{0}+X_{1}$, makes sense and for $t>0$ we can consider the parametrized family of penalty problems given by

$$
K(t, x ; \vec{X})=\inf \left\{\left\|x_{0}\right\|_{X_{0}}+t\left\|x_{1}\right\|_{X_{1}}: x=x_{0}+x_{1}, x_{i} \in X_{i}, i=0,1\right\} .
$$

To see the effect of the penalty $t$ let us suppose, for example, that $X_{1} \subset X_{0}$, with $\|x\|_{X_{0}} \leq\|x\|_{X_{1}}$. If $t$ is "very large", say $t>1$, then for every $x \in X_{0}$ the spliting $x=x+0$ "wins" and we see that $K(t, x ; \vec{X})=\|x\|_{X_{0}}$, while on the other hand, if $x \in X_{1}$, we see that $\lim _{t \rightarrow 0} \frac{K(t, x ; \vec{X})}{t}=\|x\|_{X_{1}}$. Intermediate spaces $\vec{X}_{\theta, q}$ are constructed by specifying suitable control on the decay of $K(t, x ; \vec{X})$. A typical construction (Lions-Peetre) can be described as follows: for $\theta \in(0,1), 1 \leq q \leq \infty$, let $\vec{X}_{\theta, q}=\left\{x \in \Sigma(\vec{X}):\|x\|_{\vec{X}_{\theta, q}}<\infty\right\}$, with

$$
\|x\|_{\vec{X}_{\theta, q}}=\left\{\begin{array}{cc}
\left\{\int_{0}^{\infty}\left(t^{-\theta} K(t, x ; \vec{X})\right)^{q} \frac{d t}{t}\right\}^{1 / q} & \text { if } q<\infty, \\
\sup _{t>0}\left\{t^{-\theta} K(t, x ; \vec{X})\right\} & q=\infty
\end{array} .\right.
$$

Following [15] we see that if we write

$$
x=D_{0}(t) x+D_{1}(t) x, D_{i}(t) x \in X_{i},
$$

for an optimal decomposition of $x$ for the calculation of (3.1), then

$$
K(t, x ; \vec{X})=\left\|D_{0}(t) x\right\|_{X_{0}}+t\left\|D_{1}(t) x\right\|_{X_{1}},
$$

and with suitable interpretation for the derivatives

$$
\begin{gathered}
\left\|D_{0}(t) x\right\|_{X_{0}}=K(t, x)-t \frac{d}{d t} K(t, x ; \vec{X}) \text { a.e. } \\
\left\|D_{1}(t) x\right\|_{X_{1}}=\frac{d}{d t} K(t, x ; \vec{X}) \text { a.e. }
\end{gathered}
$$

The pair $\left(L^{1}\left(\mathbb{R}^{n}\right), L^{\infty}\left(\mathbb{R}^{n}\right)\right)$ is well understood (cf. [5]). Without loss of generality we can assume that $f=|f|$. An optimal decomposition is then given by

$$
D_{0}(t)|f|=f_{f^{*}(t)}, D_{1}(t)|f|=\left(|f|-f_{f^{*}(t)}\right),
$$

where for $t>0$,

$$
f_{f^{*}(t)}= \begin{cases}|f(x)|-f^{*}(t) & \text { if } f^{*}(t)<|f(x)| \\ 0 & \text { if }|f(x)| \leq f^{*}(t)\end{cases}
$$

\footnotetext{
${ }^{15}$ For example, this will happen if $X_{1} \subset X_{0}$, with a continuous embedding.
} 
Therefore the following elementary formulae holds (cf. [5])

$$
\begin{gathered}
K\left(t, f ; L^{1}\left(\mathbb{R}^{n}\right), L^{\infty}\left(\mathbb{R}^{n}\right)\right)=\int_{0}^{t} f^{*}(s) d s=t f^{* *}(t), \\
\frac{d}{d t}\left(K\left(t, f ; L^{1}\left(\mathbb{R}^{n}\right), L^{\infty}\left(\mathbb{R}^{n}\right)\right)\right)=f^{*}(t) .
\end{gathered}
$$

In this case (3.2) and (3.3) take the form

$$
\left\|D_{0}(t) f\right\|_{L^{1}}=t f^{* *}(t)-t f^{*}(t), t\left\|D_{1}(t) f\right\|_{L^{\infty}}=t f^{*}(t) .
$$

The interaction of gradients and truncation can be quantified here by

$$
\begin{aligned}
\left\|\nabla\left(f_{f^{*}(t)}\right)\right\|_{L^{1}} & =\int_{\left\{|f|>f^{*}(t)\right\}}|\nabla| f|| d x \\
& \leq \int_{0}^{t}(\nabla|f|)^{*}(s) d s \\
& =K\left(t,|\nabla| f|| ; L^{1}, L^{\infty}\right) .
\end{aligned}
$$

Using the optimality and the definition of the penalty method (3.1) we readily get the following * reiteration* estimate (cf. [10])

$$
K\left(s, D_{0}(t) f, \vec{X}\right) \geq K(s, f)-s \frac{d}{d s} K(s, f), \quad s \leq t \quad \text { (a.e.). }
$$

As a consequence we find

$$
\left\|D_{0, \vec{X}}(t) f\right\|_{\vec{X}_{\theta, q}} \geq \int_{0}^{t}\left[\left(K(s, f ; \vec{X})-s \frac{d}{d s} K(s, f ; \vec{X})\right) s^{-\theta}\right]^{q} \frac{d s}{s}, q<\infty
$$

and

$$
\left\|D_{0, \vec{X}}(t) f\right\|_{\vec{X}_{\theta, \infty}} \geq t^{-\theta}\left[K(t, f ; \vec{X})-t \frac{d}{d t} K(t, f ; \vec{X})\right] .
$$

Let us see this method in action. We start by rewriting the weak GagliardoNirenberg inequality using

$$
\begin{aligned}
\|f\|_{M\left(L^{n^{\prime}}\right)} & =\sup _{t>0}\left\{f^{* *}(t) t^{1 / n^{\prime}}\right\} \\
& =\|f\|_{\left(L^{1}\left(R^{n}\right), L^{\infty}\left(R^{n}\right)\right)_{1 / n, \infty}} .
\end{aligned}
$$

Therefore, for $f \in L i p_{0}$, we have

$$
\|f\|_{\left(L^{1}\left(R^{n}\right), L^{\infty}\left(R^{n}\right)\right)_{1 / n, \infty}} \preceq\|\nabla f\|_{1} .
$$

Inserting the optimal decomposition we get

$$
\left\|D_{0}(t) f\right\|_{\left(L^{1}\left(R^{n}\right), L^{\infty}\left(R^{n}\right)\right)_{1 / n, \infty}} \preceq\left\|\nabla D_{0}(t) f\right\|_{1} .
$$

The left hand side of (3.8) can be estimated using (3.7) and we see that

$$
\left\|D_{0}(t) f\right\|_{\left(L^{1}\left(R^{n}\right), L^{\infty}\left(R^{n}\right)\right)_{1 / n, \infty}} \geq t^{-1 / n}\left[t f^{* *}(t)-t f^{*}(t)\right] .
$$

To estimate the right hand side of (3.8) we use (3.4) and we find

$$
\left\|\nabla D_{0}(t) f\right\|_{1} \leq t|\nabla f|^{* *}(t) .
$$

Altogether we have the familiar

$$
t^{-1 / n}\left[f^{* *}(t)-f^{*}(t)\right] \preceq|\nabla f|^{* *}(t) .
$$


If we start with the strong form of the Gagliardo-Nirenberg inequality, which in terms of interpolation norms we rewrite as,

$$
\|f\|_{\left(L^{1}\left(R^{n}\right), L^{\infty}\left(R^{n}\right)\right)_{1 / n, 1}} \preceq\|\nabla f\|_{1},
$$

we can then proceed as above. The only change is that the lower estimate is now obtained using (3.6) and we find

$$
\int_{0}^{t}\left[f^{* *}(s)-f^{*}(s)\right] s^{-1 / n} d s \preceq t|\nabla f|^{* *}(t) ;
$$

a result first derived in $\mathbf{1 7}$.

The corresponding inequalities for Log Sobolev inequalities can be obtained in analogous manner (cf. [10]) but using as starting point

$$
\|f\|_{L(\log L)^{1 / 2}\left(R^{n}, \gamma_{n}\right)} \preceq\|\nabla f\|_{L^{1}\left(R^{n}, \gamma_{n}\right)} .
$$

Finally for the connection with the theory of Burkholder-Gundy [8] note that the commutation of the gradient with truncations

$$
\left|\nabla f_{t_{1}}^{t_{2}}\right|=|\nabla f| \chi_{\left\{t_{1}<|f|<t_{2}\right\}}
$$

has the following analog in terms of Square martingale operators,

$$
S\left({ }^{\nu} f^{\tau}\right) \leq I(\nu<\tau) S(f),
$$

where $\nu, \tau$ are stopping times, and where $I$ stands for indicator function. This can be implemented to show an analogue of (3.4). Consider now the known inequality

$$
\|M f\|_{1} \preceq\|S f\|_{1},
$$

where $M$ is the maximal martingale operator. The method above then gives the following inequality due to Herz 14

$$
(M f)^{* *}(t)-(M f)^{*}(t) \preceq(S f)^{* *}(t) .
$$

We refer to $1 \mathbf{1 0}$ for more details.

Acknowledgement We are very grateful to the referee for comments and corrections that allowed us to improve the paper.

\section{References}

[1] D. Bakry, T. Coulhon, M. Ledoux and L. Saloff-Coste, Sobolev inequalities in disguise, Indiana Univ. Math. J. 44 (1995) 1033-1074.

[2] F. Barthe, P. Cattiaux, and C. Roberto, Interpolated inequalities between exponential and Gaussian, Orlicz hypercontractivity and isoperimetry. Rev. Mat. Iberoamericana 22 (2006), 993-1067.

[3] J. Bastero, M. Milman and F. Ruiz, A note on $L(\infty, q)$ spaces and Sobolev embeddings, Indiana Univ. Math. J. 52 (2003), 1215-1230.

[4] J. Bastero, M. Milman and F. Ruiz, On the Connection between Weighted Norm Inequalities, Commutators and Real Interpolation, Mem. Amer. Math. Soc. 154, American Mathematical Society, 2001.

[5] C. Bennett and R. Sharpley, Interpolation of Operators, Academic Press, Boston (1988).

[6] S. G. Bobkov and C. Houdré, Some connections between isoperimetric and Sobolev type inequalities, Mem. Amer. Math. Soc. 129 (1997), no 616.

[7] S. G. Bobkov and B. Zegarlinski, Distributions with slow tails and ergodicity of Markov semigroups in infinite dimensions, in A. Laptev (ed), Around the research of Vladimir Maz'ya I: Function Spaces, Springer, 2010, pp 13-79. 
[8] D. L. Burkholder and R. F. Gundy, Extrapolation and interpolation of quasi-linear operators on martingales, Acta Math. 124 (1970), 249-304.

[9] T. Coulhon, Heat kernel and isoperimetry on non-compact Riemannian manifolds, Contemporary Mathematics 338 (2003), 65-99.

[10] M. Cwikel, B. Jawerth and M. Milman, A note on extrapolation of inequalities, preprint, 2010.

[11] H. Federer and W. H. Fleming, Normal and integral currents, Ann. of Math. 72 (1960), 458-520.

[12] A. Garsia and E. Rodemich, Monotonicity of certain functionals under rearrangements, Ann. Inst. Fourier (Grenoble) 24 (1974), 67-116.

[13] P. Hajlasz, Sobolev inequalities, truncation method, and John domains, Papers in Analysis, Rep. Univ. Jyväskylä Dep. Math. Stat. 83, Univ. Jyväskylä, Jyväskylä, 2001, pp 109-126.

[14] C. Herz, An interpolation principle for martingale inequalities, J. Funct. Anal. 22 (1976), $1-7$.

[15] B. Jawerth and M. Milman, Interpolation of Weak Type Spaces, Math. Zeitschrift 201 (1989), 509-519.

[16] M. Ledoux, Isopérimétrie et inégalitées de Sobolev logarithmiques gaussiennes, C. R. Acad. Sci. Paris 306 (1988), 79-92.

[17] J. Martín, M. Milman and E. Pustylnik, Sobolev Inequalities: Symmetrization and Self Improvement via truncation, J. Funct. Anal. 252 (2007), 677-695.

[18] J. Martín and M. Milman, Isoperimetry and symmetrization for logarithmic Sobolev inequalities, J. Funct. Anal. 256 (2009), 149-178.

[19] J. Martín and M. Milman, Pointwise symmetrization inequalities for Sobolev functions and applications, Adv. Math 225 (2010), 121-199.

[20] J. Martín and M. Milman, Symmetrization methods in the theory of Sobolev inequalities and applications, Lecture Notes, in preparation.

[21] V. G. Maz'ya, Classes of domains and imbedding theorems for function spaces, Dokl. Acad. Nauk SSSR 3 (1960), 527-530, (Engl. transl. Soviet Math. Dokl. 1 (1961), 882-885.)

[22] V. G. Maz'ya, p-conductivity and theorems on imbedding certain functional spaces into a C-space, Dokl. Akad. Nauk SSSR 140 (1961), 299-302, (Engl. transl. Soviet Math. Dokl., 2 (1961) 1200-1203.)

[23] V. G. Maz'ya, Sobolev Spaces, Springer-Verlag, New York, 1985.

[24] E. Milman, A converse to the Maz'ya inequality for capacities under curvature lower bound, in A. Laptev (ed), Around the research of Vladimir Maz'ya I: Function Spaces, Springer, 2010, pp 321-348.

[25] E. Milman, On the role of Convexity in Isoperimetry, Spectral-Gap and Concentration, Invent. Math. 177 (2009), 1-43.

[26] E. Milman, On the role of convexity in functional and isoperimetric inequalities, Proc. London Math. Soc. 99 (2009), 32-66.

[27] B. Muckenhoupt, Weighted norm inequalities for the Hardy maximal function, Trans. Amer. Math. Soc. 165 (1972), 207-226.

[28] B. Muckenhoupt, Hardy's inequalities with weights, Studia Math 44 (1972), 31-38.

Department of Mathematics, Universitat Autònoma de Barcelona

E-mail address: jmartin@mat.uab.cat

Department of Mathematics, Florida Atlantic University, Boca Raton, Fl. 33431

E-mail address: extrapol@bellsouth.net

URL: http://www.math.fau.edu/milman 